\title{
PREDICTION OF SELF-MONITORING COMPLIANCE: APPLICATION OF THE THEORY OF PLANNED BEHAVIOUR TO CHRONIC ILLNESS SUFFERERS
}

\author{
Conor Mc Guckin ${ }^{1}$, Garry Prentice ${ }^{2}$, Christopher Mc Laughlin ${ }^{2}$, Emma Harkin ${ }^{2}$ \\ ${ }^{1}$ School of Education, Trinity College Dublin, Dublin 2, Ireland. \\ ${ }^{2}$ School of Psychology, Dublin Business School, Dublin 2, Ireland.
}

Running head: Prediction of self-monitoring compliance

Key Words: chronic obstructive pulmonary disease (COPD), diabetes, asthma, Theory of Planned Behaviour (TPB)

Editorial Correspondence:

Conor Mc Guckin, School of Education, Trinity College Dublin, Dublin 2, Ireland. Telephone: 00 (353) 871300177, Fax: 00 (353) 16777238, E-mail:

conor.mcguckin@tcd.ie 


\begin{abstract}
Chronic obstructive pulmonary disease (COPD), diabetes, and asthma are chronic illnesses that affect a substantial number of people. The continued high cost of clinic and hospital based care provision in these areas could be reduced by patients self-monitoring their condition more effectively. Such a move requires an understanding of how to predict selfmonitoring compliance. Ajzen's (1991) Theory of Planned Behaviour (TPB) makes it possible to predict those clients who will comply with medical guidelines, prescription drug intake, and self-monitoring behaviours (peak flow or blood sugar levels). Ninety-seven clients attending a medical centre located in a large urbanized area of Northern Ireland completed TPB questionnaires. Significant amounts of variance explained by the TPB model indicated its usefulness as a predictor of self-monitoring behaviour intentions in the sample. The results also highlighted the importance of subjective norm and perceived behavioural control within the TPB in predicting intentions. The utility of the TPB in this study also provides evidence for health promotion professionals that costly clinic / hospital treatment provision can be reduced, whilst also being satisfied with ongoing client self-monitoring of their condition.
\end{abstract}




\section{INTRODUCTION}

The cost of ill health affects the health care system, the individuals and their families. An increased onus on individual self-monitoring of their own condition could reduce health care system costs by reducing the need to provide direct care in a clinic setting. Thus there is a need to effectively engage with, and motivate, individual self-monitoring compliance. The current study examines factors related to self-monitoring compliance in relation to three of the most chronic illnesses in Ireland: Chronic obstructive pulmonary disease (COPD), diabetes, and asthma.

There is a need to combat the rising costs of ill health, especially costs arising from COPD, diabetes, and asthma. One way to reduce costs and evidence better health related outcomes would be to predict compliance rates of medical treatment, specifically selfmonitoring behaviour. This would more reliably inform health care professionals in their selection of patients who would be more likely to fully engage in effective selfmonitoring behaviours. This approach would have a double cost benefit in that it would (i) result in increased health of the individual, and (ii) reduce clinic / hospital admissions. To predict compliance rates, one can usefully apply psychological models, such as the Theory of Reasoned Action (TRA: Ajzen \& Fishbein, 1980) and the extended Theory of Planned Behaviour (TPB: Ajzen, 1991).

The TPB includes both cognitive and attitudinal influences involved in the performance of behaviour, and main predictor of behaviour is an individual's behavioural intention. This behavioural intention is seen as the proximal determinant of behaviour, as the more one intends to engage in a particular behaviour; the more likely they will follow through (Armitage, Norman, \& Conner, 2002). Contained within TPB model are three 
conceptually independent variables which directly determine intention; attitude, subjective-norm, and perceived behavioural control (PBC) (Ajzen, 1991). Attitudes refer to the level to which an individual has a favourable or unfavourable evaluation of the behaviour. Subjective-norm is the individual's estimate of the extent to which 'significant others' want them to perform the behaviour (Ajzen, 1991; Ajzen \& Fishbein, 1980). Lastly, PBC was added to the original Theory TRA model to allow for the prediction of behaviours, which were non-volitional. PBC not only examines the perceived ease or difficulty in performing the behaviour, but also past experiences, and perceived obstacles (Ajzen, 1991).

Within the model there are three indirect factors; attitudinal beliefs, normative beliefs, and control beliefs (Ajzen, 1991). Attitudes serve as a function of the individuals' behavioural beliefs, which are seen as the perceived outcomes of the behaviour. Subjective-norm is the function of normative beliefs that reflect the perceptions of relevant others. Lastly, $\mathrm{PBC}$ is the function of the control beliefs that are the beliefs concerning the necessary recourses needed to carry out behaviour.

The TPB is conceived of as the attitude of one's health directly linked to their behaviour (Ajzen \& Madden, 1986). Individual attitude towards health can be influenced by perceptions of condition severity and management. This model enhances prediction of client intentions to self-monitor health-related behaviours. Through the application of the TPB, it may be possible to predict those clients who will comply with medical guidelines, prescription drug intake, and self-monitoring behaviour.

The TPB has previously been applied in many health-related studies, such as exercise (Gatch and Kendzierski's, 1990), condom use among students (Sutton, McVey, 
and Glanz, 1999), drug use (Connor, Sherlock, and Orbell, 1998), dietary change (Povey et al., 2000), and self monitoring of blood glucose levels with patients with type 1 diabetes. The current study explores the utility of the TPB in enhancing the prediction of client intention to self monitor in relation to COPD, diabetes, and asthma.

As evident from previous research (e.g., Godin, Valois, \& LaPage, 1993), the TPB model demonstrates a high predictive power in regards to intentions. Despite the current study's restriction to prediction of self-monitoring intentions, a meta-analysis by Webb and Sheeran (2006) has shown that a medium to large change in intentions still leads to at least a small to medium change in behaviour. Thus, an explanation of factors related to self-monitoring behaviour intentions can give useful insights into understanding self-monitoring behaviour compliance rates in a chronic disease population. Such insights can provide useful information for choice of interventions to improve self-monitoring compliance rates. 


\section{METHOD}

\section{Sample}

The survey was administered to 97 clients attending a medical centre located in a large urbanized area of Northern Ireland. Of the 97 clients, 23 were attending due to illnesses related to breathing difficulties (COPD and / or asthma), and 74 were attending due to diabetes related illnesses. There were 47 males and 50 females. The average age was 57 years $(S D=14.78)$

\section{Procedure}

Participants were approached over a two-month period and invited to take part in the study. Those that agreed were sent the questionnaire in the mail, with a stamp addressed envelope for questionnaire return. Those who found the completion of the questionnaire difficult were referred to the practice nurse for assistance.

\section{Measures}

Two questionnaires were developed to measure those constructs contained within the TPB (Ajzen, 1985, 2002). Each questionnaire was directly comparable, except for the change in the self-monitoring behaviour, either peak flow (COPD / asthma) or blood sugar levels (diabetes). As outlined by Ajzen (2002), all predictors were assessed directly and indirectly on a set of semantic differential scales and they were compatible with intention, based on action, context, target, and time elements. Instructional examples were provided concerning the use of the seven-point scale. Each section of the questionnaire is described below. 


\section{Attitude Toward the Behaviour}

The direct measure required respondents to assess affective feelings towards selfmonitoring on a set of four semantic differential scale items. These included feeling good / bad, nauseated /enjoyable, awful / nice, and pleasant / unpleasant. An alpha correlation value of .71 was obtained. With regards to the indirect measure, two attributes of selfmonitoring were selected in order to provide a belief measure of attitude. These were: (i) knowing what your peak flow / blood sugar levels are, and (ii), being able to deal with peak flow / blood sugar levels accordingly. Firstly, respondents were asked to evaluate each outcome on a seven-point 'good / bad' semantic differential dimension and then asked to assess their 'belief strength'. These behavioural belief statements were constructed by aligning each of the two outcomes to attitudes toward self-monitoring behaviour, and by doing so each respondent was asked to rate their best estimate of the probability of the occurrence of each consequence if they were to self-monitor. Each belief value was multiplied by its corresponding outcome value in order to obtain a composite variable of attitudinal belief. An alpha correlation value of .74 was obtained.

\section{Subjective Norm}

The direct measure of subjective norm $(\mathrm{SN})$ required respondents to rate their general level of how important 'significant others' in general would expect them to behave in regards to self-monitoring. Respondents were asked to evaluate the following statement: 'It is likely that most people who are important to you think that you should self-monitor your blood sugar / peak flow levels twice a day at home?' The measure of normative 
beliefs assessed respondents' motivation to comply with the expectations of six referents including: spouse-partner, General Practitioner, nurse, family. The summed product was calculated by each normative expectative multiplied by the motivation to comply with each referent. These six composite items obtained an alpha correlation value of .82 .

\section{Perceived Behavioural Control}

The PBC construct was measured by six items, including: 'Do you think you have the ability to self-monitor your blood sugar / peak flow twice a day at home?' An alpha correlation value of .76 was reported. Control beliefs were measured by asking respondents directly to evaluate the level of their control about self-monitoring. Eight items were posed, including: 'Complications caused by your high blood sugar levels encourage you to self-monitor your blood sugar levels / peak flow twice a day at home'. An alpha correlation of .70 was obtained.

\section{Intention}

This construct was assessed using three statements posed at separate points within the questionnaire, requiring respondents to think about the likelihood of them self monitoring twice a day. For example: 'How likely is it that you would intend to self monitor your blood sugar levels twice a day at home?' An alpha correlation of .69 was obtained. 


\section{RESULTS}

Table 1 compares the diabetic and the COPD / asthma sufferers on average rated importance of each of the two outcomes of self-monitoring behaviour. Knowing levels related to each condition did differentiate between the sufferers - those with COPD / asthma being significantly more positive about this compared to the diabetics $(t(71.69)=$ $-2.17, p<.05)$. Considering that the most positive level for outcome evaluations and behavioural belief statements was 7 , both groups of sufferers were generally positive in relation to the two outcomes of self-monitoring.

\section{$<$ Insert Table 1 here $>$}

The mean normative beliefs and motivations to comply are shown in Table 2. Both groups of sufferers indicated that all six of the referents, with the exception of neighbours, were seen as similarly likely to be in favour of self-monitoring. In addition, motivations to comply were not significantly different in relation to each referent when comparing the diabetes and COPD / asthma sufferers. The most important person, denoted by the highest normative belief and motivation to comply scores, was the nurse who would have demonstrated the self-monitoring equipment, and would have had direct contact with the sufferer groups.

$<$ Insert Table 2 here $>$ 
The mean control beliefs are shown in Table 3. The statistical analysis indicated a significant difference between sufferer groups in relation to fear of negative side effects. Thus, COPD/ Asthma sufferers were significantly more likely to be discouraged compared to diabetics $(t(27.45)=2.27, p<.05)$. The seriousness of the COPD / asthma participants' condition was also significantly more likely to discourage their selfmonitoring behaviour compared to the diabetics $(t(92)=2.02,, p<.05)$. Considering that the most positive level for control beliefs was 7 , both groups of sufferers were generally positive in relation to the seven factors that might prevent or encourage selfmonitoring, with the exception of the differences outlined above.

\section{$<$ Insert Table 3 here $>$}

The mean TPB direct measures are shown in Table 4. These indicate that both groups of sufferers had similar average levels in relation to each component of the TPB. For example, the groups were just as likely to be in favour or against self-monitoring (attitude) irrespective of whether they were a diabetes or COPD / asthma sufferer. Taking into account the highest possible level for each component, both groups were generally positive in relation to the TPB direct measures, with the exception of the relatively moderate average attitude level.

$<$ Insert Table 4 here $>$ 
Zero-order correlations between the TPB variables are shown in Table 5. The strongest relationship with self-monitoring intentions is with the normative beliefs variable, followed by PBC measured directly. The role of significant others to the individual and their attitudes towards self-monitoring were significantly related to their self-monitoring intentions. In relation to the direct measures of attitudes towards self-monitoring, subjective norm and their perceived level of control over self-monitoring behaviour, all were significantly related to their corresponding indirect belief based measures.

$<$ Insert Table 5 here $>$

\section{The Prediction of Intentions to Self-Monitor}

As suggested by Ajzen (1985), two regression analyses were separately conducted. In the first, self-monitoring intentions were regressed on the direct measures of attitudinal belief towards self-monitoring and normative belief. At stage 2, control belief was added, thus implementing the testing of the TPB model. In the second regression analysis, the direct equivalent of the TPB variables were chosen (See Table 6).

\section{$<$ Insert Table 6 here $>$}

The results of these regression analyses highlight the importance of normative belief as a determinant of intentions to self-monitor. More specifically, in relation to the indirect measures, normative belief is the only variable that has a significant impact on intentions to self-monitor. Taking into account the non-significant $R_{2}$ increase from stage 1 to stage 
2 (the $R_{2}$ increase was $.02, p>.05$ ) the normative belief variable provided the largest contribution within the significant amount of variance $\left(R_{2}=.32\right)$ explained and multiple correlation coefficient $(R=.56)$ of the TPB indirect model $(F(3,66)=10.10, p<.01)$. Also represented in Table 6 are the results of the second regression analysis, in which intentions were regressed on the direct measures of attitude and subjective norm (SN). The significant impact of SN is still apparent in the TRA model and to a slightly lesser degree in the TPB model. SN provides the greatest contribution within the significant amount of variance explained $\left(R_{2}=.33\right)$ and multiple correlation coefficient $(R=.57)$ of the TRA direct model. The addition of perceived behavioural control (PBC) in the second stage resulted in a significant increase in the amount of variance explained (the $R_{2}$ increase was $.13, p<.01$ ), with PBC providing the largest contribution within the significant amount of variance explained $\left(R_{2}=.46\right)$ and multiple correlation coefficient $(R$ $=.68)$ of the TPB direct model $(F(3,48)=13.43, p<.01)$. 


\section{DISCUSSION}

The significant amount of variance explained by this TPB model indicates that it was a useful predictor of self-monitoring behaviour intentions. This study also highlights the importance of subjective norm and perceived behavioural control in predicting intention.

The more the individual intends to engage in a particular behaviour, the more likely they will carry out that behaviour according to the TPB (Webb \& Sheeran, 2006). Thus it is important to identify factors that predict intentions. In relation to the indirect measures, normative beliefs had a significant impact on behaviour. The direct measures showed the importance of perceived behavioural control alongside subjective norm, where the variance explained significantly increased providing support for the TPB compared to TRA. The importance of subjective norm compared to the relative lack of impact of attitudes in relation to intentions contradicts previous research, which frequently indicates a lesser role for subjective norm (Armitage \& Conner, 2001; Hagger, Chatzisarantis, \& Biddle, 2002). In fact, Ajzen's (1991) review of 19 TPB studies found that in nearly every case significant relationships between attitudes and intentions occurred, while significant subjective norm-intentions relationships only occurred about half the time. Firstly, this suggests that any interventions encouraging self-monitoring behaviour effectiveness and maintenance should take into account the role of significant others and perceived behavioural control issues. Secondly, the role of attitudes in selfmonitoring behaviour may be limited and despite positive underlying beliefs in relation to self-monitoring behaviour, does not relate to any greater intentions to self-monitor compared to those with less positive beliefs. 
It was expected that the more positive one's attitude is towards self-monitoring behaviour outcomes, the more likely the respondent will want to carry out such selfmonitoring behaviour. This expectation was reinforced by the findings of this study. There were positive beliefs shown by many chronic disease suffers within the sample for knowing self- monitoring levels and being able to deal with these levels.

However, the COPD / asthma sufferers had significantly more positive attitudes in regard to knowing about their condition than the diabetic sufferers. The high level of behavioural beliefs found in this study supports research conducted by Ajzen (1991), that if a healthy outcome of the illness is possible, there is more of a chance that the individual will carry out the health related behaviour.

Normative beliefs were investigated as well as the motivations to comply for both diabetes and COPD / asthma sufferers. All six of the referents, with the exception of neighbours, were seen as similarly likely to be in favour of self-monitoring irrespective of whether they had diabetes or COPD / asthma. The Diabetic group scored the nurse to be the person who most expected them to continue with the self-monitoring behaviour. The COPD / asthma group found their General Practitioner (GP) to be the referent who would most wanted them to continue with the self-monitoring behaviour, closely followed by their nurse. The Diabetics motivation to comply with self-monitoring behaviour was mostly influenced by their nurse whereas the COPD / asthma group self-monitoring behaviour was mostly influenced by their GP. These positive normative beliefs and motivation to comply perceptions are encouraging as the nurse and GP are the individuals involved in explaining the need to self-monitor and use of appropriate equipment to facilitate self-monitoring. This suggests that the health professionals involved in this 
study communicated the self-monitoring information in an understandable way, while getting across the importance of the activity (Ahmad et al., 2010).

The control beliefs of were compared, with the COPD / asthma sufferers being significantly more likely to be discouraged to continue self-monitoring due to fear of the negative side effects than the Diabetic sufferers. Similarly, a significant difference was found among the COPD / asthma sufferers who were more likely to be discouraged to continue to self-monitor if the condition was serious. These issues could be further addressed in any information provided by GPs and nurses in clinics where patient selfmonitoring of COPD/ asthma and diabetes is provided.

The findings of this study should be examined while taking into account some limitations. Firstly, it was not possible to follow-up on self-monitoring behaviours but as indicated by a meta-analysis by Webb and Sheeran (2006), a medium to large change in intentions still leads to at least a small to medium change in behaviour.

Secondly, the sample size is relatively small in the current study but the proportions of COPD / asthma and diabetics within the sample were reflective of the patients attending the clinic used in the study. The sample size did not noticeably reduce the occurrence of significant results.

The present study does show the utility of the TPB model, above the TRA in explaining intentions to self-monitor, highlighting the importance of behavioural control, alongside subjective norm, in predicting self-monitoring intentions. The positive influence of the health professionals in this study is encouraging in relation to the future effectiveness of the self-monitoring programme provided at the clinic. The greater likelihood of discontinuance of self-monitoring in the asthma / COPD group due to the 
Prediction of Self-monitoring Compliance 16

seriousness of their condition is something that should be supervised in this and similar self-monitoring programmes. 


\title{
REFERENCES
}

\begin{abstract}
Ahmad, A., Hugtenburg, J., Welschen, L. M. C., Dekker, J.M. \& Nijpels, G. (2010).
Effect of medication review and cognitive behaviour treatment by community pharmacists of patients discharged from the hospital on drug related problems and compliance: design of a randomized controlled trial. BMC Public Health, 10, 133.
\end{abstract}

Ajzen, I. (1985). From intentions to actions: A theory of planned behavior. In J. Kuhl \& J. Beckman (Eds.), Action-control: From cognition to behavior (pp. 11- 39). Heidelberg, Germany: Springer.

Ajzen, I. (2002). Constructing a TPB questionnaire: Conceptual and methodological considerations (Retrieved September 12, 2010, from http://www.people.umass.edu/aizen/pdf/tpb.measurement.pdf).

Ajzen, I. (1991). The theory of planned behaviour. Organisational Behaviour and Human Decision Processes, 50, 179-211.

Ajzen, I., \& Fishbein, M. (1980). Understanding attitudes and predicting social behavior. Englewood Cliffs, NJ: Prentice-Hall. 
Ajzen, I., \& Madden, T. J. (1986). Prediction of goal-directed behavior: attitudes, intentions, and perceived behavioral control. Journal of Experimental Social Psychology, $22,453-474$.

Armitage, C. J., \& Conner, M. (2001). Efficacy of the Theory of Planned Behaviour: A meta-analytic review. British Journal of Social Psychology, 40, 471-499.

Armitage, C. J., Norman, P., \& Conner, M. (2002). Can the theory of planned behaviour mediate the effects of age, gender and multidimensional health locus of control? British Journal of Health Psychology, 7, 299-316.

Conner, M., Sherlock, K., \& Orbell, S. (1998). Psychosocial determinants of ecstasy use in young people in the UK. British Journal of Health Psychology, 3, 295-317.

Gatch, C. L., \& Kendzierski, D. (1990). Predicting exercise intentions: the theory of planned behavior. Research Quarterly for Exercise and Sport, 61, 100-102.

Godin, G., Valois, P., \& LePage, L. (1993). The pattern of influence of perceived behavioural control upon exercising behaviour: An application of Ajzen's theory of planned behaviour. Journal of Behavioural Medicine, 16, 81- 102. 
Hagger, M. S., Chatzisarantis, N. \& Biggle, S. J. H. (2002). A meta-analytic review of the theories of reasoned action and planned behavior in physical activity: An examination of predictive validity and the contribution of additional variables. Journal of Sport and Exercise Psychology, 24, 3-32.

Povey, R., Conner, M., Sparks, P., James, R., \& Shepherd, R. (2000). Application of the Theory of Planned Behaviour to two dietary behaviours: Roles of perceived control and self-efficacy. British Journal of Health Psychology, 5, 121-139.

Sutton, S., McVey, D., \& Glanz, A. (1999). A comparative test of the theory of reasoned action and the theory of planned behaviour in the prediction of condom us intentions in a national sample of English young people. Health Psychology, 18, 72-81.

Webb, T. L., \& Sheeran, P. (2006). Does changing behavioural intentions engender behavior change? A meta-analysis of the experimental evidence. Psychological Bulletin, 132, 249-268. 
Table 1. Mean behavioural beliefs and outcome evaluations: diabetes and COPD / asthma sufferers compared

\begin{tabular}{|c|c|c|c|c|}
\hline \multirow[b]{2}{*}{$\begin{array}{l}\text { Outcomes of self- } \\
\text { monitoring twice a day at } \\
\text { home }\end{array}$} & \multicolumn{2}{|c|}{$\begin{array}{l}\text { Outcome } \\
\text { evaluations }\end{array}$} & \multicolumn{2}{|c|}{$\begin{array}{c}\text { Behavioural belief } \\
\text { statements }\end{array}$} \\
\hline & diabetes & $\begin{array}{l}\text { COPD / } \\
\text { asthma }\end{array}$ & diabetes & $\begin{array}{l}\text { COPD / } \\
\text { asthma }\end{array}$ \\
\hline 1. Knowing levels & 6.07 & $6.45^{*}$ & 6.60 & 6.43 \\
\hline $\begin{array}{l}\text { 2. Being able to deal with } \\
\text { levels }\end{array}$ & 5.96 & 5.95 & 6.43 & 6.35 \\
\hline
\end{tabular}

*Significantly different at $p<.05 ;{ }^{* *}$ significantly different at $p<.01$.

Note: For all items a seven-point scaling procedure was used ranging from 'extremely good/likely' (7) to 'extremely bad/unlikely' (1). 
Table 2. Mean normative beliefs and motivations to comply: diabetes and COPD / asthma sufferers compared

\begin{tabular}{|c|c|c|c|c|}
\hline \multirow[b]{2}{*}{ Referents } & \multicolumn{2}{|c|}{ Normative beliefs } & \multicolumn{2}{|c|}{ Motivation to comply } \\
\hline & diabetes & $\begin{array}{l}\text { COPD / } \\
\text { asthma }\end{array}$ & diabetes & $\begin{array}{l}\text { COPD / } \\
\text { asthma }\end{array}$ \\
\hline 1. Spouse/Partner & 6.20 & 5.22 & 5.92 & 4.94 \\
\hline 2. General Practitioner & 6.18 & 6.16 & 6.46 & 6.55 \\
\hline 3. Neighbours & 4.16 & 3.11 & 3.77 & 3.00 \\
\hline 4. Nurse & 6.51 & 6.05 & 6.58 & 6.25 \\
\hline 5. Family & 6.13 & 5.10 & 6.00 & 5.45 \\
\hline 6. Other sufferers & 5.67 & 5.10 & 4.84 & 4.60 \\
\hline
\end{tabular}

*Significantly different at $p<.05 ; * *$ significantly different at $p<.01$.

Note: $\quad$ For all items a seven-point scaling procedure was used ranging from

'extremely likely' (7) to 'extremely unlikely' (1). 
Table 3. Mean control beliefs: diabetes and COPD / asthma sufferers compared

\begin{tabular}{lcc}
\hline Factors that might prevent or & \multicolumn{2}{c}{ Control beliefs } \\
\cline { 2 - 3 } encourage self-monitoring & Diabetes & COPD / asthma \\
\cline { 2 - 3 } 1. Complications caused by & 6.20 & 6.60 \\
condition & & $4.48^{*}$ \\
2. Fear of negative side effects & 5.72 & 4.52 \\
3. Modification of lifestyle & 5.01 & 5.76 \\
4. Medication for condition & 5.63 & $4.38^{*}$ \\
5. Condition is serious & 5.47 & 4.62 \\
6. On holidays & 4.68 & 4.95 \\
7. Having visitors/relations & 5.28 & \\
\hline
\end{tabular}

*Significantly different at $p<.05 ; * *$ significantly different at $p<.01$.

Note: $\quad$ For all items a seven-point scaling procedure was used ranging from

'extremely positive/not discouraging' (7) to 'extremely

negative/discouraging' (1). 
Table 4. Mean theory of planned behaviour direct measures: diabetes and COPD / asthma sufferers compared.

\begin{tabular}{lcc}
\hline & \multicolumn{2}{c}{ TPB direct measures } \\
\cline { 2 - 3 } & diabetes & COPD / asthma \\
\hline 1. Attitude & 16.64 & 18.15 \\
2. SN & 6.19 & 5.76 \\
3. PBC & 37.70 & 38.63 \\
4. Intention & 18.07 & 17.25 \\
\hline
\end{tabular}

*Significantly different at $p<.05 ; * *$ significantly different at $p<.01$.

Key: $\quad$ SN: Subjective Norm; PBC: Perceived Behavioural Control

Note: $\quad$ Highest possible scores for: Attitudes (28), SN (7), PBC (42), and Intention (21). 
Table 5. Zero-order correlations between selected variables

\begin{tabular}{|c|c|c|c|c|c|c|c|}
\hline & 1 & 2 & 3 & 4 & 5 & 6 & 7 \\
\hline 1. Intention & - & & & & & & \\
\hline 2. Attitude & $.33^{*}$ & - & & & & & \\
\hline \multirow[t]{2}{*}{ 3. Attitudinal beliefs } & .11 & & - & & & & \\
\hline & & $.37^{* *}$ & & & & & \\
\hline \multirow[t]{2}{*}{ 4. SN } & & .21 & $.24^{*}$ & - & & & \\
\hline & $.49^{* *}$ & & & & & & \\
\hline \multirow[t]{2}{*}{ 5. Normative Beliefs } & & .16 & .05 & & - & & \\
\hline & $.61 * *$ & & & $.50 * *$ & & & \\
\hline \multirow[t]{2}{*}{ 6. $\mathrm{PBC}$} & & & & & & - & \\
\hline & $.52 * *$ & $.51^{* *}$ & $.38^{* *}$ & $.56^{* *}$ & $.32 * *$ & & \\
\hline 7. Control Beliefs & .19 & $.30^{*}$ & $.26^{*}$ & .14 & .04 & $.27 *$ & - \\
\hline
\end{tabular}

*Significant at $p<.05 ; * *$ significant at $p<.01$

Key: $\quad$ SN: Subjective Norm; PBC: Perceived Behavioural Control 
Table 6. Regression of intention on attitude, subjective norm and perceived behavioural control

$\mathrm{R} \quad R^{2} \quad \mathrm{~b}$

Indirect measures

Stage 1: TRA

.55

$.30^{* *}$

Attitudinal beliefs

.14

Normative beliefs

$.52 * *$

Stage 2: TPB

.56

$.32^{* *}$

Attitudinal beliefs

.09

Normative beliefs

$.52^{* *}$

Control beliefs

.14

\section{Direct measures}

Stage 1: TRA

.57

$.33^{* *}$

Attitude

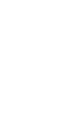

.24

SN

.68

$.46^{* *}$

Stage 2: TPB

.04

Attitude

$.29 *$

PBC

$.47^{* *}$

*Significant at $p<.05 ; * *$ Significant at $p<0.01$

Key: $\quad$ SN: Subjective Norm; PBC: Perceived Behavioural Control 